\title{
Emergence Versus Self-Organisation: Different Concepts but Promising When Combined
}

\author{
Tom De Wolf and Tom Holvoet \\ Department of Computer Science, Kuleuven, \\ Celestijnenlaan 200A, 3001 Leuven, Belgium \\ \{Tom.DeWolf, Tom.Holvoet\}@cs.kuleuven.ac.be
}

\begin{abstract}
A clear terminology is essential in every research discipline. In the context of ESOA, a lot of confusion exists about the meaning of the terms emergence and self-organisation. One of the sources of the confusion comes from the fact that a combination of both phenomena often occurs in dynamical systems. In this paper a historic overview of the use of each concept as well as a working definition, that is compatible with the historic and current meaning of the concepts, is given. Each definition is explained by supporting it with important characteristics found in the literature. We show that emergence and self-organisation each emphasise different properties of a system. Both phenomena can exist in isolation. The paper also outlines some examples of such systems and considers the combination of emergence and self-organisation as a promising approach in complex multi-agent systems.
\end{abstract}

\section{Introduction}

In the context of engineering self-organising applications there are two very important concepts to consider: emergence and self-organisation. In many multiagent systems and complex adaptive systems in general, a combination of the two concepts is often used. As a consequence, much literature describes emergence and self-organisation incorrectly as synonyms and this results in misconception about their meaning. When engineering such applications, using a clear terminology is very important. To clarify the distinction between emergence and self-organisation, this paper's goal is to propose a working definition of both concepts. This definition is supported by characteristics that most literature describes as essential for emergence or self-organisation.

Emergence and self-organisation each emphasise very different characteristics of a system's behaviour. Both phenomena can exist in isolation and they can co-exist in a dynamical system. The first two sections of this paper describe each phenomenon separately by giving a historic overview of the use of each concept, proposing a working definition, and outlining their important characteristics to explain and support the definition given. The third section relates emergence and self-organisation to each other by discussing their similarities and differences.

S. Brueckner et al. (Eds.): ESOA 2004, LNCS 3464, pp. 1,15 2005.

(C) Springer-Verlag Berlin Heidelberg 2005 
This is illustrated with examples where each phenomenon occurs separately. After that, a section is devoted to the combination of both phenomena in a single system. Finally we conclude this paper.

\section{Emergence}

Typically, people describe 'emergence' as the phenomenon where global behaviour arises from the interactions between de local parts of the system. In most literature there is nothing more than this vague description. Examples of emergence around us are: global pheromone paths that arise from local pathfollowing and pheromone-dropping ants, the swarming movement of a flock of birds, a traffic jam from the interactions of cars, etc.

The goal of this section is to develop a more detailed working definition for 'emergence'. First, a historic overview of the early use of the concept is given. The second part proposes a definition of emergence that is consistent with the given history and outlines the important characteristics found in literature.

\subsection{Historic Overview}

Emergence is not a new topic [1] 1 . Conceptual constructs such as 'whole before its parts' (i.e. to consider an explanation in terms of the global behaviour more important than explaining how the system works in terms of local behaviour) and 'Gestalt' (i.e. a configuration or pattern of elements so unified as a whole that it cannot be described merely as a sum of its parts), which resemble emergence, can be found in western thought since the time of ancient Greeks.

However, 'whole before its parts' and 'Gestalt' refer to a pre-given coherent entity, whereas emergence is not pre-given but a dynamical construct arising over time. In the context of a dynamical system, the meaning of emergence is not new either. It was used over 100 years ago by the English philosopher G.H. Lewes in 1875. Lewes distinguished between 'resultant' and 'emergent' chemical compounds coming about from a chemical reaction [2]:

(...) although each effect is the resultant of its components, we cannot always trace the steps of the process, so as to see in the product the mode of operation of each factor. In the latter case, I propose to call the effect an emergent. It arises out of the combined agencies, but in a form which does not display the agents in action (...). (italics added)

Lewes' term was borrowed during the 1920s to form the backbone of a loosely joined movement in the sciences, philosophy and theology known as emergent evolutionism or proto-emergentism [1]. The concept of emergence was hotly debated and mainly used against reductionism, which stated that a system can be reduced to the sum of its parts. Proto-emergentism had few answers when it came to understanding how emergence itself was possible, i.e. how the lower-level inputs are transformed to the higher-level outputs during emergence.

\footnotetext{
${ }^{1}$ The historic overview of emergence is based on 1 .
} 
A second movement, called neo-emergence or complexity theory [1, tries to address the lack of understanding emergence. The concept of emergence in complex systems has very diverse scientific and mathematical roots: cybernetics, solid state/ condensed matter physics, evolutionary biology, artificial intelligence, artificial life, etc. There are actually four central schools of research that each influences the way emergence in complex systems is studied:

- Complex adaptive systems theory, which became famous at the Santa Fe Institute and which explicitly uses the term 'emergence' to refer to the macro-level patterns arising from interacting agents (see [3], [4], and [5]);

- Nonlinear dynamical systems theory and Chaos theory, which promulgates the central concept of attractors, i.e. a specific behaviour to which the system evolves. One kind of attractor is the so called strange attractor that the philosopher of science David Newman (1996) [6] classifies as an authentically emergent phenomenon.

- The synergetics school, which initiated, among others, the study of emergence in physical systems. They describe the idea of an order parameter that influences which macro-level coherent phenomena a system exhibits [7].

- Far-from-equilibrium thermodynamics, which was introduced by Ilya Prigogine and which refers to emergent phenomena as dissipative structures arising at far-from-equilibrium conditions $[8]$.

In short, the uses of the concept of emergence refer to two important characteristics: a global behaviour that arises from the interactions of the local parts, and that global behaviour cannot be traced back to the individual parts.

\subsection{A Working Definition}

It is important that the concept of emergence is used consistently in literature. In the first place we need to be consistent with the historic use of the concept, as outlined above. In current literature, this is not such a big problem w.r.t. emergence. There is a larger misconception about the meaning of self-organisation, which is discussed later. The definition that we propose as a working definition for emergence is:

A system exhibits emergence when there are coherent emergents at the macro-level that dynamically arise from the interactions between the parts at the micro-level. Such emergents are novel w.r.t. the individual parts of the system.

The definition above uses the concept of an 'emergent' as a general term to denote the result of the process of emergence: properties, behaviour, structure, patterns, etc. The 'level' mentioned refers to certain points of view. The macrolevel considers the system as a whole and the micro-level considers the system from the point of view of the individual entities that make up the system.

This definition resulted from an extensive literature study, which identified the most important characteristics found in literature. The remainder of this part outlines these characteristics in order to explain the different aspects of the proposed definition in more detail. 
Micro-Macro effect $[3,9,10,1,11,12,13,14,15,16,17,18,19,20,21]$. This is the most important characteristic and is mentioned explicitly in most literature. A micro-macro effect refers to properties, behaviours, structures, or patterns that are situated at a higher macro-level and arise from the (inter)actions at the lower micro-level of the system. We call such properties 'emergents'. In other words, the global behaviour of the system (i.e. the emergent) is a result from the interactions between the individual entities of the system.

Radical Novelty $[9,11,1,22,17,19,10,20,21,13]$. The global behaviour is novel w.r.t. the individual behaviours at the micro-level, i.e. the individuals at the micro-level have no explicit representation of the global behaviour. In terms of reductionism this is formulated as: the macro-level emergents are not reducible to the micro-level parts of the system (= non-reductionism). In literature there are various formulations: 'not directly described by' 9, 10, 'can not be reduced to' [1], 'neither predictable nor deducible from' 1], 'without reference to the global pattern' [17, 'the whole is greater than the sum of its parts' 13.

From [22] we learn that we must pay attention. Stating that emergents are not captured by the behaviour of the parts is a serious misunderstanding. Radical novelty arises because the collective behaviour is not readily understood from the behaviour of the parts. The collective behaviour is, however, implicitly contained in the behaviour of the parts if they are studied in the context in which they are found. Emergent properties cannot be studied by physically taking a system apart and looking at the parts (=reductionism). They can, however, be studied by looking at each of the parts in the context of the system as a whole.

Coherence $[1,14,13,12,22,16]$. Coherence refers to a logical and consistent correlation of parts. Emergents appear as integrated wholes that tend to maintain some sense of $i d e n t i t y$ over time (i.e. a persistent pattern). Coherence spans and correlates the separate lower level components into a higher level unity, i.e. correlations between components are needed to reach a coherent whole 22]. This coherence is also called 'organisational closure' 12 .

Interacting Parts [13, 17, 18, 14, 12]. The parts need to interact - parallelism is not enough. Without interactions, interesting macro-level behaviours will never arise. The emergents arise from the interactions between the parts.

Dynamical $[1,12,3,13,17,10,20$. In systems with emergence, emergents arise as the system evolves in time. Such an emergent is a new kind of behaviour that becomes possible at a certain point in time. Therefore, as a dynamical construct we can relate the appearance of emergents to the appearance of new attractors in dynamical systems, i.e. bifurcations [1, 12.

Decentralised Control [13, 12, 16]. Decentralised control is using only local mechanisms to influence the global behaviour. There is no central control, i.e. no single part of the system directs the macro-level behaviour. The actions of the 
parts are controllable. The whole is not directly controllable. This characteristic is a direct consequence of the radical novelty that is required for emergence. Centralised control is only possible if that central part of the system has a representation of the global behaviour (e.g. a plan).

Two-Way Link [13, 20, 21]. In emergent systems there is a bidirectional link between the macro-level and the micro-level. From the micro-level to the macrolevel, the parts give rise to an emergent structure (see 'micro-macro effect' above). In the other direction, the emergent structure influences its parts. Higher level properties have causal effects on the lower level, i.e. downward causation. For example, path-formation with ants: the emergent path influences the movement of the micro-level ants because they follow the pheromones.

Robustness and Flexibility [13, 12]. The need for decentralised control and the fact that no single entity can have a representation of the global emergent, implies that such a single entity cannot be a single point of failure. Emergents are relatively insensitive to perturbations or errors. Increasing damage will decrease performance, but degradation will be 'graceful': the quality of the output will decrease gradually, without sudden loss of function. The failure or replacement of a single entity will not cause a complete failure of the emergent. This flexibility makes that the individual entities can be replaced, yet the emergent structure can remain. For example, birds in a flock or cars in a traffic jam can be replaced by other birds or cars, yet the flock and traffic jam phenomena remain.

\section{Self-Organisation}

An intuitive and linguistic definition of self-organisation given by Dempster in 1998 23] is: "Self-organisation refers to exactly what is suggested: systems that appear to organise themselves without external direction, manipulation, or control." The 'organisation' is related to an increase in the structure or order of the system behaviour. Like the section about emergence, this section develops a more detailed working definition. An example of self-organisation is: ad-hoc networks that autonomously built their structure as network devices detect each other's presence.

\subsection{Historic Overview}

[Consider] what would happen in a new world, if God were now to create somewhere in the imaginary spaces matter sufficient to compose one, and were to agitate variously and confusedly the different parts of this matter, so that there resulted a chaos as disordered as the poets ever feigned, and after that did nothing more than lend his ordinary concurrence to nature, and allow her to act in accordance with the laws which He had established ... . I showed how the greatest part of matter of this chaos must, in accordance with these laws, dispose and arrange itself in such a way as to present the appearance of heavens; how in the meantime some of its parts must compose an earth and some planets and comets, and others a sun and fixed stars.(René Descartes, 1637 [24], part 5) 
The notion of spontaneous, dynamically-produced organisation is very old 2 . This is illustrated in the quotation above from 24, which captures the essence of self-organisation. The phenomenon is only called "self-organisation" in the years after the Second World War, in communities connected with cybernetics and computing machinery [26, 27]. The first appearance of the term seems to be in a 1947 paper by W. Ross Ashby 28].

Remarkably, Ashby gave a pretty clear explanation of what he meant by 'organisation': the organisation of a system is the functional dependence of its future state on its present state and its external inputs, if any. Ashby understood a system to be self-organising if the system changed its own organisation, rather than being changed by an external entity. Ashby's description closely matches what we will define as self-organisation later.

The main research domains, where self-organisation was studied after its introduction, were physics, computer science, and systems theory. In the physical sciences self-organisation was extensively applied, from the 1970s onwards, to pattern formation 29] and spontaneous symmetry breaking [30] and to cooperative phenomena [31]. There has been confusion about what self-organisation actually is. For example, 32 claimed that the transition from lamellar to turbulent flow is an instance of self-organisation. Others have just as vigorously denied this. There has been no resolution of the controversy, and no means to resolve it 33 . In any case, just like with emergence there is confusion about the meaning of "self-organisation".

Within computer science, the primary applications have been to learning 34 26 ; to adaptation 35; and to "emergent" or distributed computation 36, 37. Also in economics [38, 39, and in ecology [40, 17, self-organisation has begun to feature, complete with the now-expected disputes about whether certain processes are self-organising.

In the 1980s, self-organisation became one of the ideas, models and techniques bundled together as the "sciences of complexity" 41. This bundle has been successful at getting itself adopted by some researchers in essentially every science, so the idea of self-organisation is now used in a huge range of disciplines.

One of those disciplines is multi-agent systems. Multi-agent systems are used to model self-organising systems. Cooperation [42] and group formation 43. in multi-agent systems make the system more organised, which is done autonomously by the agents. A number of self-organising applications are realised [44, such as in networks [45, in robotics, and the self-organisation of a vocabulary between agents 46 .

\subsection{A Working Definition}

It is important that the concept of self-organisation is used consistently in literature. In the first place we need to be consistent with the historic use of the concept, as outlined above. Therefore 'autonomy' and 'increase in structure' should be included. In current literature, there is a often misconception about

\footnotetext{
${ }^{2}$ The historic overview of self-organisation is based on the Ph.D. of C.R.Shalizi 25.
} 
the meaning of self-organisation. For example, in [17, 16] the authors define selforganisation when they actually define emergence according to our definition. The definition that we propose as a working definition for self-organisation is:

Self-organisation is a dynamical and adaptive process where systems acquire and maintain structure themselves, without external control.

The 'structure' can be a spatial, temporal or functional structure. 'No external control' refers to the absence of direction, manipulation, interference, pressures or involvement from outside the system. This does not exclude data inputs from outside the system as long as these inputs are not control instructions. Note, the identification of the 'boundary' of the system is extremely important when deciding if a system is self-organising or not. It is important to specify what we consider as an external control and what not.

An extensive literature study identified the characteristics, considered important in literature. Below we outline these characteristics in order to explain the different aspects of the proposed definition in more detail.

Increase in Order $[25,47,20,12,11,45,15,19,44]$. One important characteristic of self-organisation is the 'organisation' part of the concept. 20] describes organisation as the arrangement of selected parts so as to promote a specific function. This restricts the behaviour of the system in such a way as to confine it to a smaller volume of its state space. This smaller region of state space is called an attractor. In essence, organisation can be looked at as an increase in the order of the system behaviour which enables the system to acquire a spatial, temporal, or functional structure. Note that not every system that has an increase in order needs to be self-organising. Complete autonomy of the behaviour is also needed (see below).

In 25, a more formal approach is used to define self-organisation. The author uses the notion of statistical complexity to denote the order mentioned in this paper. An increase in statistical complexity is considered a necessary condition for self-organisation. Statistical complexity measures the average amount of historical memory stored in the process. This formulation covers a number of other definitions found in literature. For example, 'the arrangement of selected parts' implies that the arrangement is a kind of historic memory of the process that becomes bigger when more and more parts are arranged.

An increase in order implies that such systems start from semi-organised or completely random initial conditions [44] (i.e. no historical memory). What is also possible is that a system behaviour becomes less ordered (i.e. looses historical memory) as a result from a change. Both situations leave room for an increase in order through the process of self-organisation.

The formulation 'as to promote a specific function' in [20] is important. A system with no order can not exhibit useful behaviour. But also a system with too much order can have this problem. It is possible that processes organise themselves into conditions so complex that no usable functionality can result from it. In other words, there can be too much historical memory. The systems 
in between, i.e. at the edge of order and chaos 48, 49, can exhibit a more flexible and organised behaviour. Therefore, self-organisation needs to find a balance between no order and too much order.

Autonomy $[25,47,20,12,11,45,17,19,44]$. Not every increase in order is self-organising. The second important characteristic of self-organisation is the absence of external control ('self'). A system needs to organise without interference from the outside. Other formulations are: 'without an external agent imposing it', 'spontaneous, i.e. not steered by an external system', 'the constraints on form (i.e. organisation) of interest to us are internal to the system', etc.

Does the lack of external control and autonomy mean that such a system can have no input at all? Of course not, in general, input is still possible as long as the inputs are no control instructions from outside the system. In other words, normal data input flows are allowed but the decision on what to do next should be made completely inside the system, i.e. the system is autonomous. For example, plugging in a $\mathrm{PnP}$ device in a computer can be considered as normal data input. A self-organising behaviour could be the autonomous configuration of drivers by the computer system. If a user has to install the drivers himself then there is no self-organisation.

The notion of 'boundary of a system' becomes very important here. To be able to say if a certain system is self-organising, we must first clearly define the boundary of the system. We need to separate the inside from the outside.

Adaptability or Robustness w.r.t. changes [1, 45, 12, 44]: In selforganising systems, robustness is used in terms of adaptability in the presence of perturbations and change. A self-organising system is expected to cope with that change and to maintain its organisation autonomously. In other words, a self-generated, adaptable behaviour is needed [1, and taking into account past experiences can be helpful 45. 44 formulates this adaptability as: "a change in the environment may influence the same system to generate a different task, without any change in the behavioural characteristics of its constituents".

This adaptability implies the need for the system to be able to exhibit a large variety of behaviours. Self-organisation requires the evolution towards a certain attractor in state space (i.e. towards a certain organised behaviour). There are different kinds of attractors, from a point attractor that allows only one behaviour, a limit cycle that allows periodic behaviour, towards a chaotic attractor that allows a very large variety of behaviours. To be adaptable, the system needs to make a selection between behaviours and at the same time consider a variety of behaviours [12. Too much variety, like the chaotic attractor, makes the system uncontrollable. Too much selection, like the point attractor, results in a system that is not flexible enough. This is related to balancing the system on the edge of order and chaos 48, 49] in order to be able to promote a specific function (see 'Increase in order'). For example, a system's initial conditions may support many functions (i.e. chaotic attractor), but there need to be selective 
pressures to focus the outcome [19]. For example, a system that has a chaotic attractor can balance its behaviour on a specific part of that attractor.

Dynamical, i.e. far-from-equilibrium [25, 1, 11, 12, 50]: An essential property of self-organisation is that it is a process. Over time, there is an increase in order, i.e. a dynamic towards more order.

Related to the required adaptability in a rapidly changing context, selforganising behaviour needs to be dynamic. Changes influence the organised structure. In order to maintain that structure, there needs to be a constant dynamic that handles these changes. In other words, the system needs to be far-from-equilibrium in order to maintain the structure. Prigogine [50] considers far-from-equilibrium as one of the mathematically deduced requirements. A far-from-equilibrium system is more fragile and sensitive to changes in the environment, but also more dynamic and capable to react.

\section{Comparing Emergence and Self-Organisation}

To summarise, the essence of emergence is the existence of a global behaviour that is novel w.r.t. the constituent parts of the system. The essence of selforganisation is an adaptable behaviour that autonomously acquires and maintains an increased order (i.e. statistical complexity, structure, ...). In this section we describe the similarities and the differences between both concepts.

\subsection{Similarities}

Because emergence and self-organisation each emphasise very different aspects of the system behaviour there are few similarities. The main similarity is that emergence and self-organisation are both dynamic processes arising over time. Both are also robust. However, emergence is robust w.r.t. the flexibility in the specific parts that cause the emergent properties (i.e. the failure of one single part will not result in a complete failure of the emergent property). Self-organisation is robust w.r.t. the adaptability to change and its ability to maintain the increased order. Having few similarities does not exclude that both concepts are related to each other. They complement each other when combined (see below).

\subsection{Differences}

The sections above show that emergence and self-organisation each emphasise different characteristics of a system. Both concepts can exist in isolation, which is discussed here. First we consider self-organisation without emergence, and then emergence without self-organisation is described and illustrated with examples.

Self-Organisation without Emergence. Figure1(a) schematically illustrates a system with self-organisation, but no micro-macro effect. There are no controls that come from outside the boundary of the system. The curved arrow 


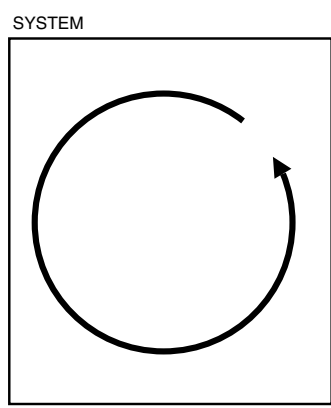

(a)

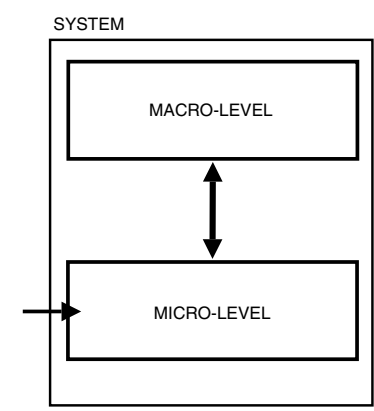

(b)

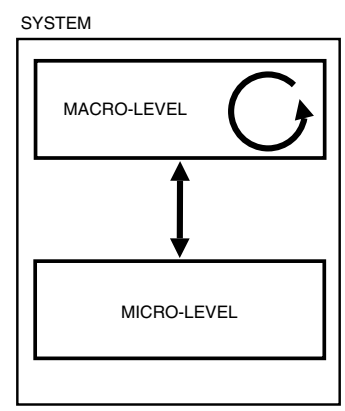

Fig. 1. (a) self-organisation without emergence; (b) emergence without selforganisation; (c) Combining Emergence and Self-Organisation

represents the internal organising process. The properties that are specific for emergence, but not needed for self-organisation, are radical novelty, micro-macro effect, flexibility w.r.t. the entities, and decentralised control. When one of these properties is not present we have no emergence.

Consider certain kinds of multi-agent systems, called a 'classical' multi-agent system in [19]. Such a system is autonomous and increases its order through interactions. However, there is no need for the system to exhibit emergent properties, i.e. properties that are novel w.r.t. the agents in the system. When, for example, every agent has a model of the global behaviour that has to be achieved, this behaviour is explicitly present in the parts of the system and thus not novel.

A system where there is a single controlling agent that directs the global behaviour (i.e. no decentralised control), needs an explicit plan in that controlling agent. Of course, a self-organising process can re-elect a controlling agent when other agents become more appropriate for the job, but there is no radical novelty.

Another important property of emergent systems is 'graceful degradation' because of the flexibility w.r.t. the entities. A single entity is not essential for the functioning of the whole system. A self-organising system where each entity is essential does not conform with the needed characteristics of emergence.

Emergence without Self-Organisation. Figure1(b) schematically illustrates the other situation. The system has a micro-macro effect, but it is not selforganising. The essential properties here are the increase in order, no external control and adaptability.

Emergence without self-organisation is definitely possible. For example in physics, thermodynamics can emerge from statistical mechanics in a stationary (and so non-self-organising) system 25. A stationary process is a process where the order is time-translation invariant, i.e. no increase in order. Consider a gas material that has a certain volume in space. This volume is an emergent property that results form the interactions (i.e. attraction and repulsion) between the individual particles. However, such a gas is in a stationary state. The statistical 
complexity remains the same over time, i.e. the particles can change place but the amount of structure remains the same. In this case, we have a system whose initial conditions are enough to exhibit emergent properties.

Adaptability refers to the need to reach a balance between selection of a specific behaviour and the consideration of a large variety of behaviours. 45 . formulates this in terms of a balance between exploration and exploitation. A system can exhibits chaos (i.e. considering a large variety of behaviours and also constantly switching between these) that emerged from the interactions between the micro-level parts. But, such a system is not self-organising because it does not organise itself to promote a specific function.

\section{Combining Emergence and Self-Organisation}

In most systems that are considered in literature, emergence and self-organisation occur together. Research in the multi-agent community and the complex adaptive systems community focuses on such systems. In very complex (multi-agent) systems, i.e. distributed, open, large, situated in a dynamic context, etc., the combination of emergence and self-organisation is recommended. In a complex (multi-agent) system there is often a need to keep the individual entities relatively simple (e.g. for scalability). Self-organisation requires an increase in order that promotes a certain function or property. Simple individuals cannot direct such a complex system, so the global coherent behaviour should emerge from the interactions between the individuals. The other way around, a complex (multiagent) systems can be required to exhibit emergent behaviour. Because of the complexity, it is impossible to impose an initial structure on such a system that results in an emergent property. The only possibility to get a coherent behaviour at the macro-level is to let that behaviour arise and organise autonomously, i.e. self-organisation. Thus, combining both phenomena is a promising approach to engineer a coherent behaviour for complex (multi-agent) systems.

Combining self-organisation and emergence in one system imposes the question on how both phenomena should be linked to each other. To answer this, there are multiple point-of-views possible. A first point of view considers selforganisation as a cause, i.e. emergent properties in complex systems are the result of a self-organising process [11, 17, 51, possibly combined with selective pressures towards a certain emergent behaviour [49. Thus, the interactions between the individual entities are the self-organisation. Self-organisation is situated at the micro-level of the emergent process. A second point of view considers self-organisation as an effect, i.e. emergence results in self-organisation. Thus, self-organisation is an emergent property. Figure 1(c) schematically illustrates what is stated in [15: "... self-organising behaviour occurs at the macro-level". This point-of-view is explained as a result of a characteristic of self-organisation, i.e. the need for an increase in order. In an emergent system, at the micro-level the dynamics are often very complicated and disordered. This means an increase in order can only occur within the global level [19, i.e. the emergents become more and more organised. The author of [19] also states that the system as a 
whole is decreasing its order. The reason for this is that at the micro-level of emergent systems the dynamics are often very complicated and disordered. Also in [25] the author states: "... self-organisation increases [statistical] complexity, while emergence, generally speaking, reduces it ...". Thus, this need for increased order seems to conform with self-organisation as an effect at the macro-level of emergence.

Because emergence and self-organisation are often described in combination with each other, a characteristic, that some authors ascribe to one of both phenomena, is probably more specific for the combination of the phenomena. This characteristic is Nonlinearity 1, 12, 50, 17): A system, without a priori order and where the emergence has to be self-organised, requires the "small cause, large effect" principle and should have an intense focus on nonlinear interactivity. Nonlinearity enables those secondary effects at the macro-level that we call emergents. This nonlinearity is often achieved through positive feedback that amplifies an initial change. The result of the first amplification again triggers positive feedback that amplifies the effect of the change. After a while, a number of components have 'aligned' themselves with the configuration created by the initial change and the configuration stops growing: the system has 'exhausted' the available resources. This alignment is often the emergent property of the system. This way, an emergent can self-organise.

Nonlinear mechanisms are related to one of the properties of self-organisation, mathematically deduced by Prigogine [50. He states that at least one of the components in the system must exhibit auto-catalysis. A system exhibits autocatalysis if one of its components is causally influenced by another component, resulting in its own increase. Actually, auto-catalysis is a kind of positive feedback (e.g. pheromone reinforcements by ants) that can cause a nonlinear effect.

In a self-organising system, the emergence should be adaptive in order to have a system that self-organises in the presence of a changing situation. When there has been a nonlinear 'alignment' with positive feedback, the only possibility to escape that alignment, and end up in a new alignment that is adapted to the new situation, is to use negative feedback. In more complex self-organising systems, there will be several interlocking positive and negative feedback loops, so that changes in some directions are amplified while changes in other directions are suppressed. In [17 the presence of positive and negative feedback is also considered important for adaptive behaviour.

\section{Conclusion}

The starting point of this paper was that it is important to use a clear terminology when engineering self-organising applications. The discussion showed that the important concepts of emergence and self-organisation refer to two distinct phenomena. They each emphasise different characteristics of a system. Confusion in literature should be avoided by using each concept correctly and certainly not as synonyms. Emergence emphasises the presence of a novel coherent macro-level emergent (property, behaviour, structure, ...) as a result from the interactions 
between micro-level parts. Self-organisation emphasises the dynamical and adaptive increase in order or structure without external control.

Both phenomena can exist in isolation, yet a combination of both phenomena is often present in complex dynamical systems. In such systems, the complexity is huge, which makes it infeasible to impose a structure a priori: the system needs to self-organise. Also, the huge number of individual entities imposes a need for emergence. For scalability we can not put an entire plan for the global structure in a single entity; we need to keep the individuals rather simple and let the complex behaviour self-organise as an emergent behaviour from the interactions between these simple entities. A combination of emergence and self-organisation, which is already applied in literature [19,44, 52, is a promising approach to engineer large-scale multi-agent systems.

This paper presents results from research sponsored by the research council of the K.U.Leuven. The results have been obtained in the Concerted Research Action on Agents for Coordination and Control - AgCo2 project.

\section{References}

1. Goldstein, J.: Emergence as a construct: History and issues. Emergence 1 (1999)

2. Lewes, G.: Problems of Life and Mind. Volume 2. Kegan Paul, Trench, Turbner, London (1875)

3. Holland, J.: Emergence: from Chaos to Order. Addison-Wesley (1998)

4. Kauffman, S.: At Home in the Universe: the Search for the Laws of SelfOrganization and Complexity. Oxford University Press (1995)

5. Langton, C.: Studying artificial life with cellular automata. In Farmer, D., Lapedes, A., Packard, N., Wendroff, B., eds.: Evolution, Games, and Learning: Models for Adaptation in Machines and Nature, Proceedings of the Fifth Annual Conference of the Center for Nonlinear Studies. (1986)

6. Newman, D.: Emergence and strange attractors. Philisophy of Science 36 (1996)

7. Haken, H.: The Science of Structure: Synergetics. Van Nostrand Reinhold, NY (1981)

8. Nicolis, G.: Physics of far-from-equilibrium systems and self-organization. In Davies, P., ed.: The New Physics. Cambridge University Press (1989)

9. Crutchfield, J.: Is anything ever new? considering emergence. Working Paper 94-03-011, Santa Fe Institute (1994)

10. Crutchfield, J.: The calculi of emergence: Computation, dynamics, and induction. Working Paper 94-03-016, Santa Fe Institute (1993)

11. Heyligen, F.: Self-organization, emergence and the architecture of complexity. In: Proceedings of the 1st European Conference on System Science, Paris (1989)

12. Heyligen, F.: The science of self-organisation and adaptivity. In: The Encyclopedia of Life Support Systems. UNESCO Publishing-Eolss Publishers (2002)

13. Odell, J.: Agents and complex systems. JOT 1 (2002) 35-45

14. Odell, J.: Objects and agents compared. JOT 1 (2002) 41-53

15. Parunak, H.V.D., Brueckner, S.: Entropy and self-organization in multi-agent systems. In: Proceedings of the Fifth International Conference on Autonomous Agents, ACM Press (2001) 124-130 
16. Engineering self-organizing applications workgroup - mission statement (2003) (preliminary version available at http://cui.unige.ch/ ${ }^{\sim}$ dimarzo/ esoawg/mission.pdf).

17. Camazine, S.: Self-Organization in biological systems. Princeton Studies in Complexity. Princeton Univ Press (2001)

18. Parunak, H., Brueckner, S., Sauter, J.: ERIM's Approach to Fine-Grained Agents. In: In Proceedings of NASA/JPL Workshop on Radical Agent Concepts (WRAC'02). (2002) (available at http://www.erim.org/ vparunak/.

19. Parunak, H.D., Brueckner, S.A.: Engineering Swarming Systems. In Bergenti, F., Gleizes, M.P., Zambonelli, F., eds.: Methodologies and Software Engineering for Agent Systems. Kluwer (2004) (to appear, available online at http://www.erim.org/ ${ }^{\sim}$ vparunak/).

20. Self-organizing systems faq. online (2003) available at http ://www.calresco.org/ sos/sosfaq.htm.

21. Lucas, C.: Emergence and evolution - constraints on form. online (2003) (available at http : //www.calresco.org/emerge.htm).

22. Bar-Yam, Y.: 0, Overview: The Dynamics of Complex Systems - examples, questions, methods and concepts. Studies in Nonlinearity. In: Dynamics of Complex Systems. Westview Press (1997)

23. Dempster, M.B.L.: A Self-Organising Systems Perspective on Planning for Sustainability. Master's thesis, University of Waterloo, School of Urban and Regional Planning (1998) (online at http://www.fes.uwaterloo.ca/u/mbldemps/pubs/).

24. Descartes, R.: Discours de la méthode pour bien conduire sa raison, et chercher la vérité dans les sciences. In: The Philosophical Writings of Descartes, 1985, Cambridge University Press. Volume I., Leiden (1637) 111-151 translated in Discours on the Method of rightly conducting one's reason and seeking truth in the sciences.

25. Shalizi, C.R.: Causal Architecture, Complexity and Self-Organization in Time Series and Cellular Automata. PhD thesis, University of Wisconsin at Madison

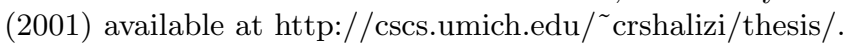

26. Yovits, M.C., Cameron, S., eds.: Self-Organising Systems: Proceedings of an Interdisciplinary Conference, Oxford, Pergamon Press (1960) vol. 2 of International Tracts in Computer Science and Technology and Their Application.

27. Von Foerester, H., Jr., G.Z.Z., eds.: Principles of Self-Organization: Transactions of the University of Illinois Symposium on Self-Organization, June 1959, New York, Information Systems Branch, U.S. Office of Naval Research, Pergamon Press (1962)

28. Ashby, W.R.: Principles of self-organizing dynamic systems. Journal of General Psychology 37 (1947) 125-128

29. Ball, P.: The Self-Made Tapestry: Pattern Formation in Nature. Oxford University Press (1999)

30. Nicolis, G., Prigogine, I.: Self-Organization in Nonequilibrium Systems: From Dissipative Structures to Order through Fluctuations. Wiley, New York (1977)

31. Haken, H.: Synergetics: An introduction: Nonequilibrium Phase Transitions and Self-Organization in Physics, Chemistry, and Biology. Springer Verlag (1977)

32. Klimontovich, Y.L.: Turbulent Motion and the Structure of Chaos: A New approach to the Statistical Theory of Open Systems. Kluwer Academic (1990/1991)

33. Frisch, U.: Turbulence: The Legacy of A.N. Kolmogorov. Cambridge University Press, Cambridge, England (1995)

34. Selfridge, O.G.: Pandemonium: A paradigm for learning. In Blake, D., Uttley, A., eds.: The Mechanisation of Thought Processes. Volume 10 of National Physical Laboratory Symposia. Her Majesty's Stationary Office, London (1959) 511-529 
35. Holland, J.H.: Adaptation in Natural and Artificial Systems: An Introductory Analysis with Applications to Biology, Control, and Artificial Intelligence. 2 edn. MIT Press, Cambridge, Massachusetts (1992) First edition in 1975.

36. Forrest, S., ed.: Emergent Computation: Self-Organizing, Collective, and Cooperative Phenomena in Natural and Artificial Computing Networks: Proceedings of the Ninth Annual International Conference of the Center of Nonlinear Studies, Los Alamos, New Mexico, 1989, Amsterdam, North Holland (1990)

37. Crutchfield, J.P., Mitchell, M., eds.: The Evolution of Emergent Computation, Proceedings of the National Academy of Sciences. Volume 92. (1995)

38. Krugman, P.R.: The Self-Organizing Economy. Blackwell, Oxford (1996)

39. Shalizi, C.R.: Review of krugman, p.r. (1996) 'the self-organizing economy'. In: The Bactra Review. Volume 11. (1996) available at

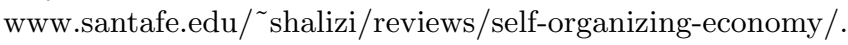

40. Arthur, W.: The Green Machine: Ecology and the Balance of Nature. Basil Blackwell, Oxford (1990)

41. Pagels, H.R.: The Dreams of Reason: The Computer and the Rise of the Sciences of Complexity. Simon and Schuster, New York (1988)

42. Steels, L.: Cooperation between distributed agents through self-organisation. In: Proceedings of the First European Workshop on Modelling Autonomous Agents in a Multi-Agent World, Elsevier Science Publishers Holland (1990) 175-196

43. Ghanea-Hercock, R.: Spontaneous group formation in multi-agent systems. In: Proceedings of Workshop on Self-Organisation in Multi-Agent Systems. (2000)

44. Mostefaoui, S.K., Rana, O.F., Foukia, N., Hassas, S., Marzo, G.D., Aart, C.V., Karageorgos, A.: Self-Organising Applications: A Survey. In: Proceedings of the International Workshop on Engineering Self-Organising Applications 2003. (2003) (extended version in 'Engineering Self-Organising Systems - Nature-Inspired Approaches to Software Engineering', ISBN: 3-540-21201-9, Springer Verlag).

45. Foukia, N., Hassas, S.: Towards self-organizing computer networks: A complex system perspective. In: Proceedings of the International Workshop on Engineering Self-Organizaing Applications 2003, Melbourne, Austrialia (2003)

46. Oudeyer, P.Y.: Self-organisation of a lexicon in a structured society of agents. In Floreano, D., Nicoud, J.D., Mondada, F., eds.: Advances in Artificial Life (ECAL 99). LNAI 1674, Berlin, Springer-Verlag (1999) 726-729

47. Haken, H.: Information and Self-Organisation: A Macroscopic Approach to Complex Systems. Springer Verlag, germany (1998)

48. Langton, C.G.: Computation at the edge of chaos: Phase transitions and emergent computation. Physica D 42 (1990) 12-37

49. Kauffman, S.: The Origins of Order: Self-Organization and Selection in Evolution. Oxford University Press (1993)

50. Glansdorff, P., Prigogine, I.: Thermodynamic study of structure, stability and fluctuations. Wiley, New York (1978)

51. Mamei, M., Zambonelli, F.: Self-organisation in multi-agent systems: a middleware approach. In: Proceedings of the International Workshop on Engineering SelfOrganising Applications 2003. (2003)

52. Hadeli, Valckenaers, P., Zamfirescu, C.B., Brussel, H.V., Germain, B.S., Holvoet, T., Steegmans, E.: Self-organising in multi-agent coordination and control using stigmergy. In: Proceedings of the International Workshop on Engineering SelfOrganising Applications 2003. (2003) 